\title{
Use of fluorescence to guide resection or biopsy of primary brain tumors and brain metastases
}

\author{
*Serge Marbacher, M.D., M.Sc., ${ }^{1,5}$ Elisabeth Klinger, M.D., ${ }^{2}$ Lucia Schwyzer, M.D., ${ }^{1,5}$ \\ IngEborg Fischer, M.D. ${ }^{3}$ Edin Nevzati, M.D. ${ }^{1}$ Michael DiePers, M.D. ${ }^{2,5}$ \\ Ulrich Roelcke, M.D., ${ }^{4,5}$ Ali-Reza Fathi, M.D., ${ }^{1,5}$ Daniel Coluccia, M.D., ${ }^{1,5}$ \\ AND JaVier FAndino, M.D. ${ }^{1,5}$
}

Departments of ${ }^{1}$ Neurosurgery, ${ }^{2}$ Neuroradiology, ${ }^{3}$ Pathology, and ${ }^{4}$ Neurology, and ${ }^{5}$ Brain Tumor Center, Kantonsspital Aarau, Aarau, Switzerland

Object. The accurate discrimination between tumor and normal tissue is crucial for determining how much to resect and therefore for the clinical outcome of patients with brain tumors. In recent years, guidance with 5-aminolevulinic acid (5-ALA)-induced intraoperative fluorescence has proven to be a useful surgical adjunct for gross-total resection of high-grade gliomas. The clinical utility of 5-ALA in resection of brain tumors other than glioblastomas has not yet been established. The authors assessed the frequency of positive 5-ALA fluorescence in a cohort of patients with primary brain tumors and metastases.

Methods. The authors conducted a single-center retrospective analysis of 531 patients with intracranial tumors treated by 5-ALA-guided resection or biopsy. They analyzed patient characteristics, preoperative and postoperative liver function test results, intraoperative tumor fluorescence, and histological data. They also screened discharge summaries for clinical adverse effects resulting from the administration of 5-ALA. Intraoperative qualitative 5-ALA fluorescence (none, mild, moderate, and strong) was documented by the surgeon and dichotomized into negative and positive fluorescence.

Results. A total of 458 cases qualified for final analysis. The highest percentage of 5-ALA-positive fluorescence in open resection was found in glioblastomas $(96 \%, \mathrm{n}=99 / 103)$. Among other tumors, 5-ALA-positive fluorescence was detected in $88 \%(n=21 / 32)$ of anaplastic gliomas (WHO Grade III), 40\% ( $n=8 / 19)$ of low-grade gliomas (WHO Grade II), no $(n=0 / 3)$ WHO Grade I gliomas, and 77\% $(n=85 / 110)$ of meningiomas. Among metastases, the highest percentage of 5-ALA-positive fluorescence was detected in adenocarcinomas $(48 \%, n=13 / 27)$. Low rates or absence of positive fluorescence was found among pituitary adenomas $(8 \%, \mathrm{n}=1 / 12)$ and schwannomas $(0 \%, \mathrm{n}=$ 0/7). Biopsies of high-grade primary brain tumors showed positive rates of fluorescence similar to those recorded for open resection. No clinical adverse effects associated with use of 5-ALA were observed. Only 1 patient had clinically silent transient elevation of liver enzymes.

Conclusions. Study findings suggest that the administration of 5-ALA as a surgical adjunct for resection and biopsy of primary brain tumors and brain metastases is safe. In light of the high rate of positive fluorescence in highgrade gliomas other than glioblastomas, meningiomas, and a variety of metastatic cancers, 5-ALA seems to be a promising tool for enhancing intraoperative identification of neoplastic tissue and optimizing the extent of resection. (http://thejns.org/doi/abs/10.3171/2013.12.FOCUS13464)

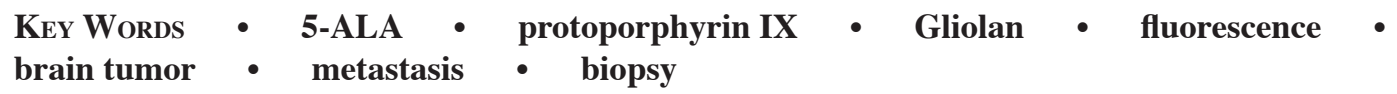

$\mathrm{E}$ XTENT of resection is an important prognostic factor that affects survival times for most patients with primary or secondary malignant brain tumors. ${ }^{22,23,34}$ However, during open tumor resection, it is often difficult to distinguish between normal brain and neoplastic tissue and to identify tumor margins, ${ }^{16,24,32}$ and during stereotactic biopsy of brain tumors, the sampling of positive pathological tissue specimens can be challenging. ${ }^{25}$ For patients with glioblastoma (WHO Grade IV), the preop-

Abbreviation used in this paper: 5 -ALA $=5$-aminolevulinic acid.

* Drs. Marbacher and Klinger contributed equally to this work. erative oral administration of 5-aminolevulinic acid (5ALA) improves the intraoperative visualization of tumor with a blue-violet light source..$^{30,38-40,45,49}$ For a series of patients with glioblastoma who underwent 5-ALA-guided tumor resection, radiologically confirmed complete resection rates of up to $83 \%$ were recently reported. 6

A growing body of evidence indicates that the extent of tumor resection and therefore overall survival times for patients with glioblastoma can be positively influenced by 5-ALA fluorescence-guided surgery. ${ }^{30,39,45,49}$ In recent years, interest has increased in the assessment of 5-ALA as a promising intraoperative adjunct for detecting nonglioblastoma high-grade glioma, low-grade 
glioma, and meningioma tissue..$^{5,43,45}$ More recently, a retrospective study of 52 patients demonstrated that $62 \%$ of brain metastases were positive for intraoperative 5-ALA fluorescence. ${ }^{20}$

Preliminary reports indicate that 5-ALA fluorescence increases the safety and accuracy of stereotactic and endoscopic brain biopsy procedures. ${ }^{25,31,47}$ It has been argued that speed and safety are increased by minimizing sampling errors. ${ }^{25,47}$

Very few cases of adverse effects associated with use of 5-ALA during glioma surgery have been reported. In a series of 74 patients the authors described adverse events (phototoxic reaction, generalized edema) for only 2 patients. ${ }^{14}$ The authors of large approval studies among 500 patients receiving 5-ALA before glioma resection have reported no adverse events associated with the drug. ${ }^{37,39}$

In this study, we analyzed our institutional experience with 458 consecutive cases of primary brain tumors and brain metastases treated either by microsurgical tumor resection or biopsy after oral administration of 5-ALA.

\section{Methods}

\section{Patient Population and Data Acquisition}

The study was supervised by the institutional review board of our institution, Kantonsspital Aarau. The off-label use of 5-ALA for intracranial tumors was approved by the Swiss Agency for Therapeutic Products (Swissmedic). Informed consent for surgical treatment and administration of 5-ALA (Gliolan, medac $\mathrm{GmbH}$ ) was obtained for all patients.

From February 2007 through December 2011, a total of 531 patients underwent 5-ALA-guided open resection or biopsy of primary brain tumors or metastases at Kantonsspital Aarau. Of these, 458 patients fulfilled the following inclusion criteria: 1) age 18 years or older, 2) radiologically confirmed intracranial mass lesion, and 3) consensus to either operate or perform a biopsy after case review by the hospital's interdisciplinary neurooncology tumor board. Clinical data were collected from patient charts, surgical consent forms, operative reports and intraoperative video recordings, report forms on intraoperative 5-ALA fluorescence, histology reports, discharge summaries, and laboratory results.

The following characteristics were further analyzed: patient age, patient sex, histological diagnosis, tumor location, intraoperative 5-ALA fluorescence positivity, type of surgical procedure (open resection or biopsy), liver function test results (liver transaminases and $\gamma$-glutamyltransferase), and adverse reactions associated with administration of 5-ALA. The grading of intraoperative qualitative 5-ALA fluorescence (none, mild, moderate, and strong) was documented by the surgeon. Because this qualitative interpretation and grading of visible fluorescence is prone to interobserver bias, we therefore dichotomized all findings into negative (none) and positive (mild, moderate, and strong) fluorescence.

\section{Clinical Management}

All patients underwent preoperative MRI with a standard examination protocol performed by a MAGNETOM Espree 1.5-T or a MAGNETOM Avanto 1.5$\mathrm{T}$ scanner (Siemens). The protocol consisted of regular 5-mm diffusion-weighted imaging and susceptibilityweighted imaging sequences, a 4-mm T2-weighted turbo spin echo sequence (TR/TE $6080 \mathrm{msec} / 96 \mathrm{msec}$ ), a 6-mm T1-weighted spin echo sequence (TR/TE $450 \mathrm{msec} / 9.5$ $\mathrm{msec}$ ), and a true inversion recovery magnitude dark-fluid fat-saturated sequence (TR/TE $9000 \mathrm{msec} / 121 \mathrm{msec}$ ), each in axial planes. Additionally, a contrast-enhanced $0.9-\mathrm{mm}$ T1-weighted volumetric interpolated breathhold examination high-resolution sequence (TR/TE 6.2 $\mathrm{msec} / 2.38 \mathrm{msec}$ ) was performed in a sagittal direction, with multiplanar reformat workups in 1-mm axial and coronal planes.

In accordance with the regulations of the European Medicines Agency (www.ema.europa.eu/), 5-ALA was administered only in the absence of any known history of liver disease and signs of significant hepatic dysfunction as seen clinically or determined by preoperative liver function tests. Further exclusion criteria for the administration of 5-ALA were history of photosensitivity, patient or family history of porphyria, or pregnancy. An aqueous solution of 5-ALA (Gliolan) was orally administered, at $20 \mathrm{mg} / \mathrm{kg}$ body weight, 3-5 hours before beginning the operation.

All open tumor resections were performed using standard microsurgical techniques and intraoperative neuronavigation tools (StealthStation Treon, Medtronic, or VectorVision, Brainlab AG). Tumor biopsy samples were obtained stereotactically using frameless neuronavigation systems (BrainPro Titanium Biopsy Cannula Set, PAJUNK GmbH) based on preoperative or intraoperative MRI scans (Polestar N20, Medtronic).

For evaluation of 5-ALA fluorescence positivity, blue-violet light was applied periodically during and at the end of tumor resection or after biopsy samples were obtained (440-nm blue-violet light source, OPMI Pentero, Carl Zeiss AG). To avoid phototoxic skin reactions from the sensitizing effect of protoporphyrin IX, patients remained in a dark environment for about 48 hours after surgery. ${ }^{9,21}$

The formalin-fixed tissue specimens underwent standard histological evaluation with $\mathrm{H} \& \mathrm{E}$ staining and immunohistochemical analysis at our institution.

Postoperatively, we discussed all patients at our interdisciplinary neurooncology tumor board. Depending on age, histologicasl result, WHO tumor grade, clinical condition, and performance status, patients either underwent treatment with standard protocols including radiotherapy with or without chemotherapy or they were observed with regular clinical and MRI follow-up assessments.

\section{Results}

A total of 531 5-ALA-guided microsurgical resections or biopsies of intracranial tumors were performed. Of those, 73 (14\%) patients were excluded from the study, leaving 458 patients for final analysis. Reasons for exclusion were missing or conflicting (in terms of 5-ALA fluorescence) operative reports, intraoperative 5-ALA report 
forms, or video recordings (for 55 patients) or missing discharge reports (for 18 patients). Details on patient selection and clinical characteristics of the study population are presented in Fig. 1 and Table 1, respectively. All histological entities and their intraoperative 5-ALA fluorescence positivity are summarized in Table 2.

Intraoperative 5-ALA fluorescence was positive in $96 \%(n=99 / 103)$ of glioblastomas, $83 \%(n=5 / 6)$ of anaplastic oligodendrogliomas, and $82 \%(n=14 / 17)$ of oligoastrocytomas (WHO Grade III) (Fig. 2). Compared with high-grade gliomas, there were only a small number of microsurgically resected low-grade gliomas (WHO Grades I and II) (Fig. 3). None of the WHO Grade I tumors (pilocytic astrocytoma, $n=0 / 2$; ganglioglioma, $n=$ 0/1) showed 5-ALA fluorescence. Fluorescence was positive in $42 \%(\mathrm{n}=5 / 12)$ of astrocytomas (WHO Grade II), $33 \%(\mathrm{n}=1 / 3)$ of oligoastrocytomas (WHO Grade II), and $100 \%(n=2 / 2)$ of oligodendrogliomas (WHO Grade II).

Meningiomas formed the largest group of nonglial tumors resected in open surgery. In $77 \%(\mathrm{n}=85 / 110)$ of all meningiomas, 5-ALA fluorescence was positive (Fig. 4). Rates of fluorescence positivity were similar among WHO Grade I meningiomas $(78 \%, \mathrm{n}=73 / 94)$ and WHO Grade II meningiomas $(71 \%, \mathrm{n}=10 / 14)$.

Overall, positive 5-ALA fluorescence was documented for $52 \%(n=34 / 65)$ of patients with brain metastases (Fig. 5). For further analysis, brain metastases were subdivided according to histology. Most metastases ( $\mathrm{n}=$ 13/27) were adenocarcinomas. The primary sites of the adenocarcinomas were lungs for 5 patients, gastrointestinal tract for 7 patients (esophagus $[\mathrm{n}=3]$, stomach $[\mathrm{n}=$ $1]$, and colon $[\mathrm{n}=3]$ ), and kidneys for 3 patients. 5-ALA fluorescence was positive in $86 \%(n=6 / 7)$ of metastases from gastrointestinal adenocarcinomas but only $20 \%$ (n
$=1 / 5)$ of metastases from adenocarcinoma of the lung. In contrast, 5-ALA fluorescence was positive in $100 \%$ ( $\mathrm{n}=$ 4/4) of metastases from small cell carcinomas of the lung. Other subtypes of metastases with high rates of positive 5-ALA fluorescence were invasive ductal carcinomas (no specific type) of the breast $(71 \%, \mathrm{n}=5 / 7)$ and melanomas $(76 \%, \mathrm{n}=3 / 4)$.

A total of 82 patients underwent stereotactic biopsy (Fig. 6). Of those, 5-ALA fluorescence was positive for $85 \%(n=44 / 52)$ of high-grade gliomas (WHO Grades III and IV). A total of 6 biopsies from diffuse large B-cell lymphomas of the CNS were performed, of which 5-ALA fluorescence was positive for half $(50 \%, \mathrm{n}=3 / 6)$.

No clinically apparent adverse effects associated with use of 5-ALA were observed. Postoperative liver function testing was performed only when liver dysfunction was suspected. For 1 female patient with postoperative abdominal discomfort, a moderate increase in liver enzymes was found and was graded as an adverse event $(0.2 \%, \mathrm{n}=1 / 458)$. Antiedema therapy with corticosteroids was continued. The patient had no known liver disease or risk factors for liver dysfunction. Phototoxicity was not documented for any of the analyzed patients. However, because of the retrospective nature of the study, we cannot exclude the possibility that mild or temporary skin irritation occurred despite strict light protection.

\section{Discussion}

The results of this retrospective analysis of 458 patients demonstrate that 5-ALA is a safe surgical adjunct to open resection and biopsy of brain tumors. Apart from high-grade gliomas, rates of 5-ALA-positive fluorescence were high for a variety of other tumor entities. To

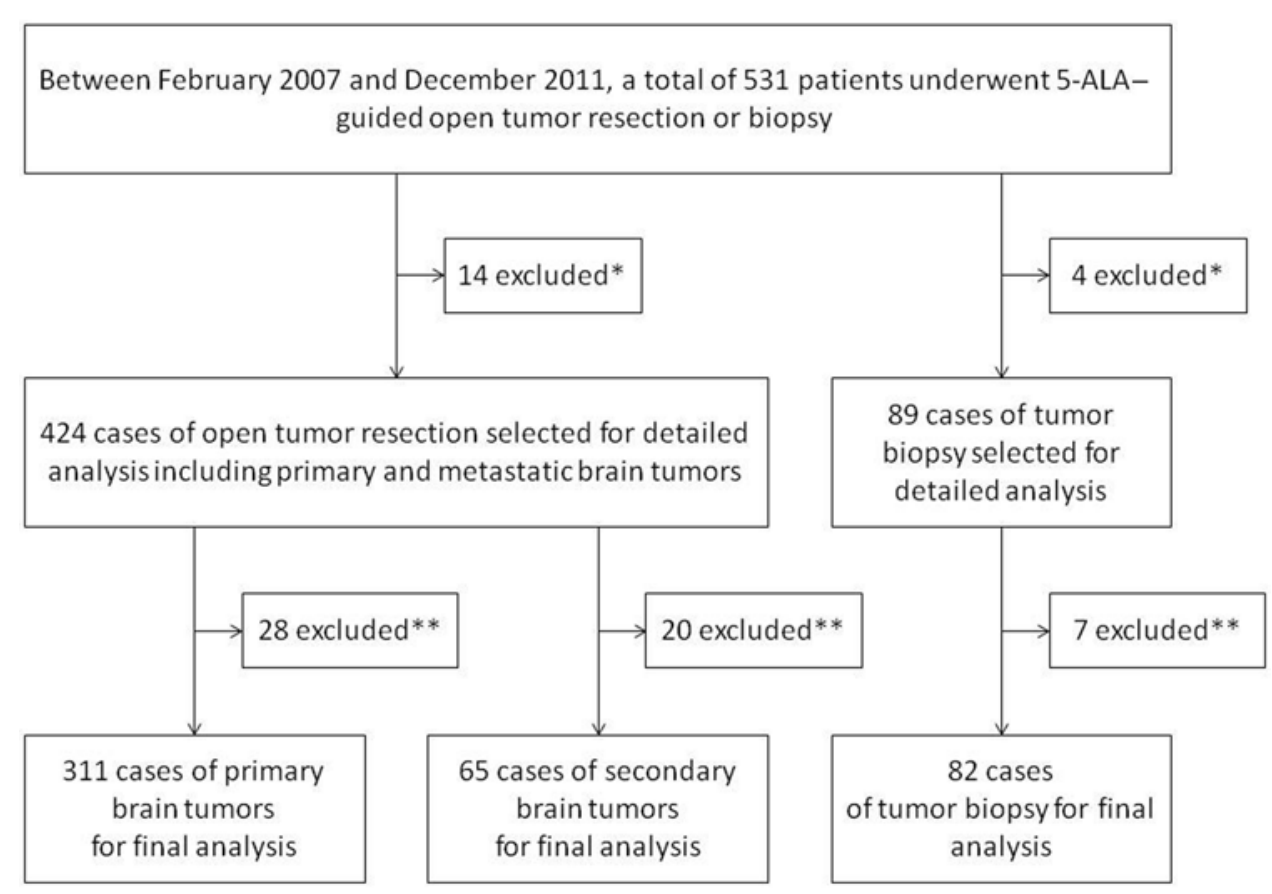

FIG. 1. Patient selection process. Asterisk indicates cases excluded because of missing data from source documents. Double asterisks indicate cases excluded because of missing or conflicting data concerning intraoperative 5-ALA fluorescence behavior. 
TABLE 1: Clinical characteristics of 458 patients*

\begin{tabular}{|c|c|}
\hline Characteristic & No. $(\%)$ \\
\hline \multicolumn{2}{|l|}{ sex } \\
\hline M & $242(52.8)$ \\
\hline $\mathrm{F}$ & $216(47.2)$ \\
\hline \multicolumn{2}{|l|}{ tumor etiology } \\
\hline primary brain tumor & $383(83.6)$ \\
\hline metastatic brain tumor & 75 (16.4) \\
\hline \multicolumn{2}{|l|}{ tumor location } \\
\hline supratentorial & $413(90.2)$ \\
\hline frontal & 156 \\
\hline parietal & 76 \\
\hline occipital & 37 \\
\hline temporal & 95 \\
\hline central region & 12 \\
\hline insular & 8 \\
\hline intraventricular & 13 \\
\hline intrasellar/suprasellar & 16 \\
\hline infratentorial & $42(9.2)$ \\
\hline cerebellopontine angle & 11 \\
\hline fourth ventricle & 4 \\
\hline brainstem & 11 \\
\hline cerebellar & 13 \\
\hline clival & 3 \\
\hline supratentorial \& infratentorial & $3(0.7)$ \\
\hline \multicolumn{2}{|l|}{ surgical procedure } \\
\hline open resection & $376(82.1)$ \\
\hline biopsy & $82(17.9)$ \\
\hline adverse events associated w/ 5-ALA & $1(0.2)$ \\
\hline
\end{tabular}

the best of our knowledge, ours is the largest study of 5-ALA fluorescence behavior in nonglioblastoma lesions.

\section{Molecular Mechanisms of 5-ALA-Induced Fluorescence}

The hydrophilic 5-ALA is enzymatically converted to photosensitive protoporphyrin IX, which is transformed to heme after insertion of iron by the enzyme ferrochelatase. ${ }^{11,28}$ The detailed molecular mechanisms of selective accumulation in some tumor cells remain obscure. Several mechanisms have been hypothesized: altered activity of ferrochelatase, iron chelators, protoporphyrin IX synthesis, increased intracellular 5-ALA uptake, or disturbances in protoporphyrin IX outflow from malignant cells., $, 8,29$

Given the hydrophilic nature of 5-ALA, disruption of the blood-brain barrier seems to be necessary for increased 5-ALA uptake into neoplastic brain lesions. Stummer et al. reported residual enhancement on MR images in 18 of 19 patients with residual intraoperative tissue fluorescence. ${ }^{38}$ Recent data demonstrating a positive correlation between quantitative measurements of ex vivo protoporphyrin IX and gadolinium concentrations in patients undergoing fluorescence-guided glioma resec- tion support the idea that blood-brain barrier breakdown is an important mechanism for increased intratumor protoporphyrin IX fluorescence. ${ }^{46}$ Although significant, the correlation coefficient in the latter study was modest, and the authors concluded that additional factors probably play a key role in accumulated levels of protoporphyrin IX. Other factors, such as tumor tissue cell density and proliferation status in gliomas, are also strong predictors of protoporphyrin IX accumulation. ${ }^{44}$

In meningioma tissue, tumor vascularization plays less of a role in intratumor 5-ALA accumulation. We recently demonstrated that meningioma fluorescence was not associated with preoperative steroid administration or peri-tumor edema. ${ }^{5}$

\section{5-ALA Fluorescence in Primary Brain Tumors}

Percentages of positive fluorescence in open resection were consistently high for high-grade gliomas (WHO Grades III and IV) $(88 \%, \mathrm{n}=122 / 138)$ and biopsy $(85 \%$, $\mathrm{n}=44 / 52$ ). These findings are in line with reports in the literature of high rates of positive 5-ALA fluorescence in high-grade gliomas (glioblastomas, anaplastic oligoastrocytomas, and anaplastic oligodendrogliomas). ${ }^{49}$

In our study, 5-ALA fluorescence was positive for $40 \%$ of low-grade gliomas (WHO Grades II). Recent studies investigating 5-ALA fluorescence in low-grade gliomas have shown positivity without use of any magnification in only $17 \%(\mathrm{n}=1 / 6)$ of patients, ${ }^{44}$ whereas a positive rate of $100 \%(\mathrm{n}=10 / 10)$ could be demonstrated with the use of confocal microscopy. ${ }^{33,35}$ Ewelt et al. ${ }^{10}$ found $7.7 \%(\mathrm{n}=1 / 13)$ and Widhalm et al ${ }^{48}$ found no focal 5-ALA fluorescence in WHO Grade II tumors $(n=0 / 8)$. The authors of smaller series reported a lack of 5-ALA fluorescence in low-grade gliomas. ${ }^{14,17,42}$ These differences can be explained in part by the subjective nature and dichotomization of 5-ALA grading. In our series, even minimal fluorescence was characterized as fluorescent positive. Other possible explanations might be intratumor heterogeneity and sampling bias.

A possible explanation for the low rate of positive fluorescence might be that anaplastic astrocytomas, by definition, do not have endothelial proliferation, whereas anaplastic oligodendrogliomas and oligoastrocytomas frequently do. Therefore, we hypothesize that the main mechanism for the low rate of positive 5-ALA fluorescence in pure anaplastic astrocytomas compared with anaplastic oligoastrocytomas (WHO Grade III) and oligodendrogliomas (WHO Grade III) might be the result of disruption of the blood-brain barrier. ${ }^{2,3}$ It has been demonstrated that microvascular density correlates with concentrations of protoporphyrin IX in biopsy samples from gliomas. ${ }^{46}$

\section{5-ALA Fluorescence in Meningiomas}

In recent years, the range for the use of intraoperative 5-ALA fluorescence has been extended to incorporate other non-high-grade primary brain tumors such as meningoepithelial tumors. ${ }^{1,5,7,26,27}$ In our series, $77 \%$ $(\mathrm{n}=85 / 110)$ of meningiomas were 5-ALA fluorescence positive. In our previous series, $94 \%$ of 33 consecutive cases of meningioma demonstrated visible fluorescence. 
Use of fluorescence to guide brain tumor resection

TABLE 2: Histological entities

\begin{tabular}{|c|c|c|}
\hline \multirow[b]{2}{*}{ Entity } & \multicolumn{2}{|c|}{ No. of Cases (\%) } \\
\hline & 5-ALA Positive & 5-ALA Negative \\
\hline \multicolumn{3}{|l|}{ primary brain tumors, open resection } \\
\hline high-grade glioma (WHO Grades III-IV) & $122(88.4)$ & $16(11.6)$ \\
\hline glioblastoma (WHO Grade IV) & $99(96.1)$ & $4(3.9)$ \\
\hline oligodendroglioma (WHO Grade III) & $5(83.3)$ & $1(16.7)$ \\
\hline oligoastrocytoma (WHO Grade III) & $14(82.4)$ & $3(17.6)$ \\
\hline astrocytoma (WHO Grade III) & $2(22.2)$ & $7(77.8)$ \\
\hline gliosarcoma (WHO Grade IV) & $2(66.7)$ & $1(33.3)$ \\
\hline low-grade glioma (WHO Grades I-II) & $8(40.0)$ & $12(60.0)$ \\
\hline oligodendroglioma (WHO Grade II) & $2(100)$ & $0(0)$ \\
\hline oligoastrocytoma (WHO Grade II) & $1(33.3)$ & $2(66.7)$ \\
\hline astrocytoma (WHO Grade II) & $5(41.7)$ & $7(58.3)$ \\
\hline pilocytic astrocytoma (WHO Grade I) & $0(0)$ & $2(100)$ \\
\hline ganglioglioma (WHO Grade I) & $0(0)$ & $1(100)$ \\
\hline meningioma & $85(77.3)$ & $25(22.7)$ \\
\hline WHO Grade I & $73(77.7)$ & $21(22.3)$ \\
\hline WHO Grade II & $10(71.4)$ & $4(28.6)$ \\
\hline WHO Grade III & $2(100)$ & $0(0)$ \\
\hline pituitary adenoma & $1(8.3)$ & $11(91.7)$ \\
\hline medulloblastoma & $0(0)$ & $2(100)$ \\
\hline schwannoma & $0(0)$ & $7(100)$ \\
\hline ependymoma & $0(0)$ & $3(100)$ \\
\hline neurocytoma & $0(0)$ & $1(100)$ \\
\hline hemangiopericytoma & $1(33.3)$ & $2(66.7)$ \\
\hline hemangioma & $0(0)$ & $2(100)$ \\
\hline gangliocytoma & $0(0)$ & $1(100)$ \\
\hline chordoma & $1(50)$ & $1(50)$ \\
\hline other & $0(0)$ & $7(100)$ \\
\hline diffuse large B-cell lymphoma & $2(100)$ & $0(0)$ \\
\hline \multicolumn{3}{|l|}{ brain metastases, open resection } \\
\hline all metastases & $34(52.3)$ & $31(47.7)$ \\
\hline adenocarcinoma & $13(48.1)$ & $14(51.8)$ \\
\hline lung & $1(20)$ & $4(80)$ \\
\hline prostate & $0(0)$ & $1(100)$ \\
\hline esophagus & $3(100)$ & $0(0)$ \\
\hline stomach & $0(0)$ & $1(100)$ \\
\hline colon & $3(100)$ & $0(0)$ \\
\hline kidney & $2(66.7)$ & $1(33.3)$ \\
\hline urothelial carcinoma & $1(33.3)$ & $2(66.7)$ \\
\hline small cell carcinoma of the lung & $4(100)$ & $0(0)$ \\
\hline invasive ductal carcinoma (no specific type) of the breast & $5(71.4)$ & $2(28.6)$ \\
\hline squamous cell carcinoma & $1(12.5)$ & $7(87.5)$ \\
\hline melanoma & $3(75)$ & $1(25)$ \\
\hline poorly differentiated carcinoma & $3(37.5)$ & $5(62.5)$ \\
\hline endometrioid carcinoma & $1(100)$ & $0(0)$ \\
\hline mucoepidermoid carcinoma & $1(100)$ & $0(0)$ \\
\hline \multicolumn{3}{|l|}{ brain tumor, stereotactic biopsy } \\
\hline high-grade glioma (WHO Grades III-IV) & $44(84.6)$ & $8(15.4)$ \\
\hline glioblastoma (WHO Grade IV) & $32(88.9)$ & $4(11.1)$ \\
\hline
\end{tabular}

(continued) 
TABLE 2: Histological entities (continued)

\begin{tabular}{lcc}
\hline & \multicolumn{2}{c}{ No. of Cases (\%) } \\
\cline { 2 - 3 } Entity & 5-ALA Positive & 5-ALA Negative \\
\hline brain tumor, stereotactic biopsy (continued) & & $8(15.4)$ \\
high-grade glioma (WHO Grades III-IV) (continued) & $44(84.6)$ & $1(50)$ \\
oligoastrocytoma (WHO Grade III) & $1(50)$ & $3(21.4)$ \\
astrocytoma (WHO Grade III) & $11(78.6)$ & $9(75)$ \\
low-grade glioma (WHO Grades I-II) & $3(25)$ & $1(100)$ \\
oligoastrocytoma (WHO Grade II) & $0(0)$ & $7(70)$ \\
astrocytoma (WHO Grade II) & $3(30)$ & $1(100)$ \\
astrocytoma (WHO Grade I) & $0(0)$ & $0(0)$ \\
ependymoma & $1(100)$ & $0(0)$ \\
germinoma & $2(100)$ & $1(100)$ \\
multiple myeloma & $0(0)$ & $1(33.3)$ \\
gliomatosis cerebri & $2(66.7)$ & $2(66.7)$ \\
other & $1(33.3)$ & $3(50)$ \\
diffuse large B-cell lymphoma & $3(50)$ & \\
\hline
\end{tabular}

A recent study of 15 patients reported 5-ALA positivity for $80 \%$ of meningioma cases. ${ }^{43}$ The overall high rate of 5-ALA positivity suggests that 5-ALA could be a useful surgical adjunct, especially in the presence of ill-defined tumor margins (bone, dura, and brain parenchyma invasion), which is seen with some high-grade meningiomas. ${ }^{26}$

\section{5-ALA Fluorescence in Brain Metastases}

Positive intraoperative 5-ALA fluorescence was documented in $52 \%(n=34 / 65)$ of patients with brain metastases. With respect to 5-ALA fluorescence behavior, brain metastases form a heterogeneous group. Slightly higher rates of positive 5-ALA fluorescence have been reported in the literature. Kamp et al. reported positive fluorescence in $62 \%$ of metastases; 23 (70\%) of 33 adenocarcinomas were positive. ${ }^{20}$ In a series of 11 metastatic brain tumors, Utsuki et al. reported 5-ALA positivity for $82 \%$ of metastases. ${ }^{41}$

Also in our series, the rate of 5-ALA fluorescence positivity in metastases from breast cancer was high (71\%, $\mathrm{n}=5 / 7$ ). 5-ALA fluorescence was positive in only 1 of 4 urothelial carcinoma metastases. Published findings on intravesical instillation for the detection of transitional cell carcinoma indicate a detection rate of $92 \% .{ }^{15}$ Although we have no true understanding for this pronounced difference in fluorescence positivity, possible explanations are as follows: the low number of urothelial carcinoma metastasis in our series $(n=4)$, the difference between biological behavior of the primary tumor and metastatic tissue, or the difference in 5-ALA administration (topical bladder instillation vs oral administration in our series). In a case series of 11 patients with breast cancer, all tissue samples from the primary tumor site were fluorescence positive. ${ }^{12}$

\section{Use of 5-ALA in Stereotactic Biopsy of Brain Tumors}

In their series on stereotactic brain tumor biopsy, von Campe et al. reported positive 5-ALA fluorescence in high-grade gliomas in all 13 patients. ${ }^{47}$ In our series,

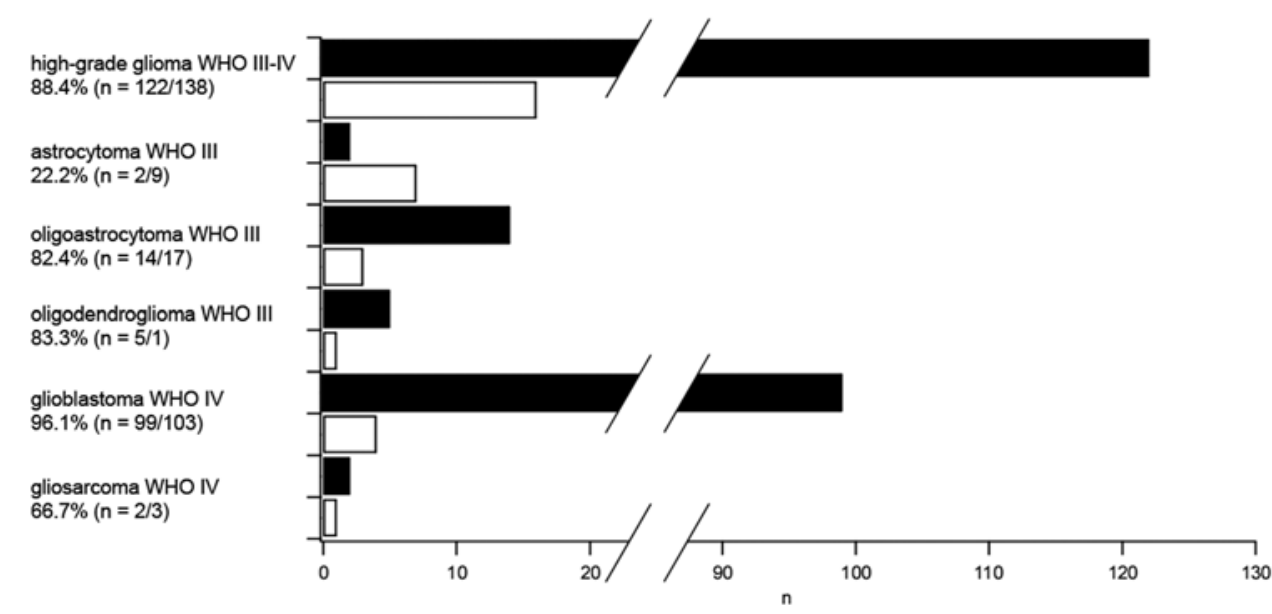

FIG. 2. Graph showing 5-ALA positivity in high-grade brain tumors. $n$ indicates cases with positive fluorescence/total number of cases; black bars = 5-ALA-positive cases; white bars = 5-ALA-negative cases. 
Use of fluorescence to guide brain tumor resection

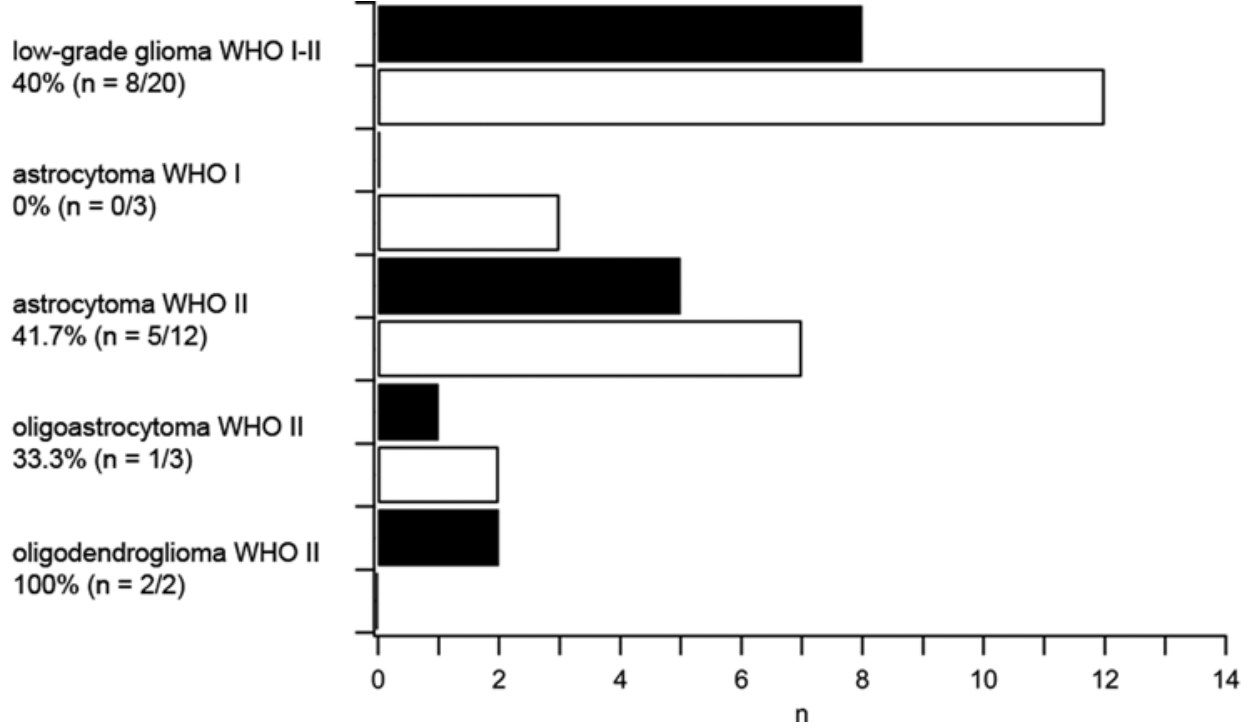

FIG. 3. Graph demonstrating 5-ALA positivity in low-grade brain tumors. $n$ indicates cases with positive fluorescence/total number of cases; black bars $=5$-ALA-positive cases; white bars $=5$-ALA-negative cases.

5-ALA fluorescence was positive in $84.6 \%(\mathrm{n}=44 / 52)$ of all biopsy samples from high-grade gliomas. On the basis of these data, we postulate that for patients with suspected high-grade glioma, the rate of negative collection of pathological tissue specimens in stereotactic biopsies can be reduced with the administration of 5-ALA.

Clinical Value of 5-ALA-Guided Resection of Primary Brain Tumors, Brain Metastases, and Biopsy of Patients With Suspected High-Grade Brain Tumors

The results of this study confirm high rates of 5-ALAinduced fluorescence in high-grade gliomas in general and in glioblastomas in particular. Intraoperative 5-ALA fluorescence was positive in $96 \%$ of glioblastomas and offered real-time in situ tumor identification, which enabled us to resect almost all neoplastic tissue in most cases. Although we cannot make a definitive statement on the positive influence of 5-ALA on the extent of tumor resection, it is becoming increasingly difficult to ignore the fact that tumor fluorescence derived from 5-ALA enables more complete resection, which leads to improved progression-free survival times for patients with malignant glioma. $30,39,45,49$ In contrast, visible 5-ALA-induced fluorescence in lowgrade gliomas (WHO Grade II) was rare, with only focal or vague fluorescence. In WHO Grade I tumors, 5-ALA fluorescence was absent. Therefore, it seems that visible 5-ALA fluorescence-guided surgery for low-grade gliomas (WHO Grade I and II) adds no value. We likewise found limited value for 5-ALA fluorescence-guided surgery for pituitary adenomas and schwannomas.

According to our observations, induced fluorescence in meningiomas is advantageous for visualizing menin-

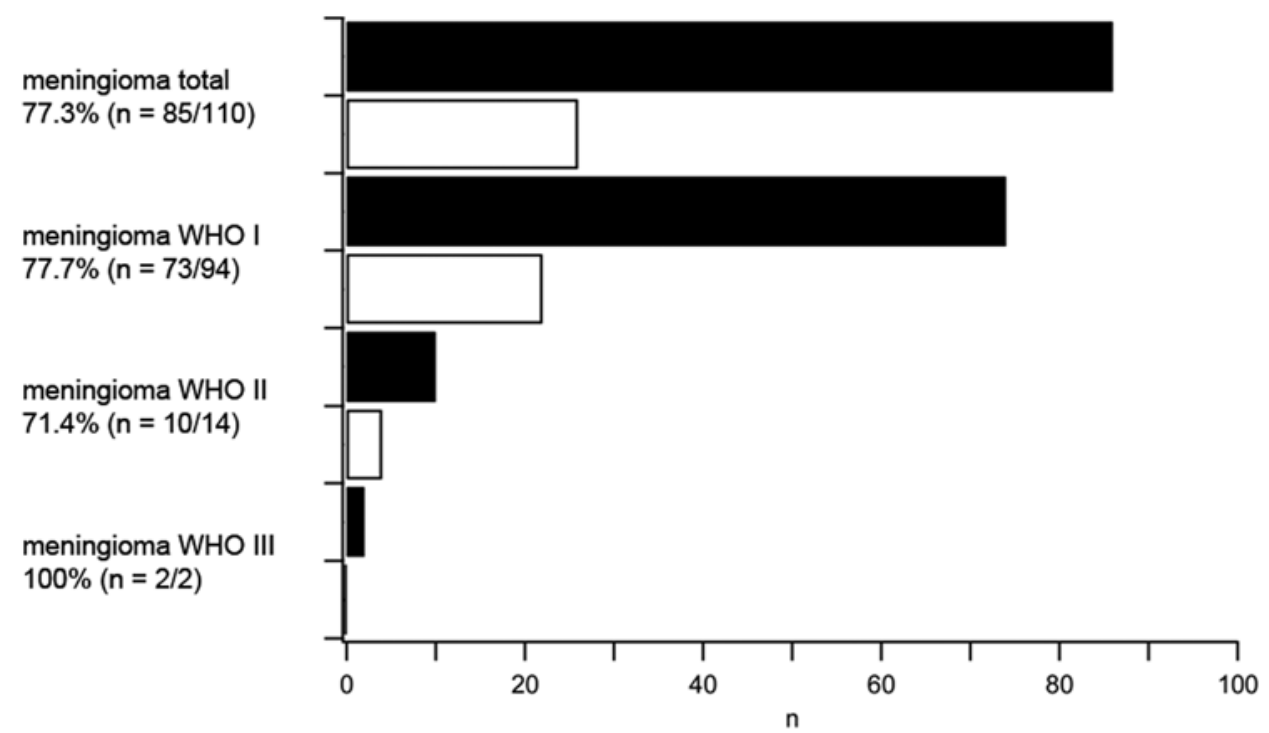

FIG. 4. Graph showing 5-ALA positivity in meningiomas. $n=$ cases with positive fluorescence/total number of cases; black bars = 5-ALA-positive cases; white bars = 5-ALA-negative cases. 


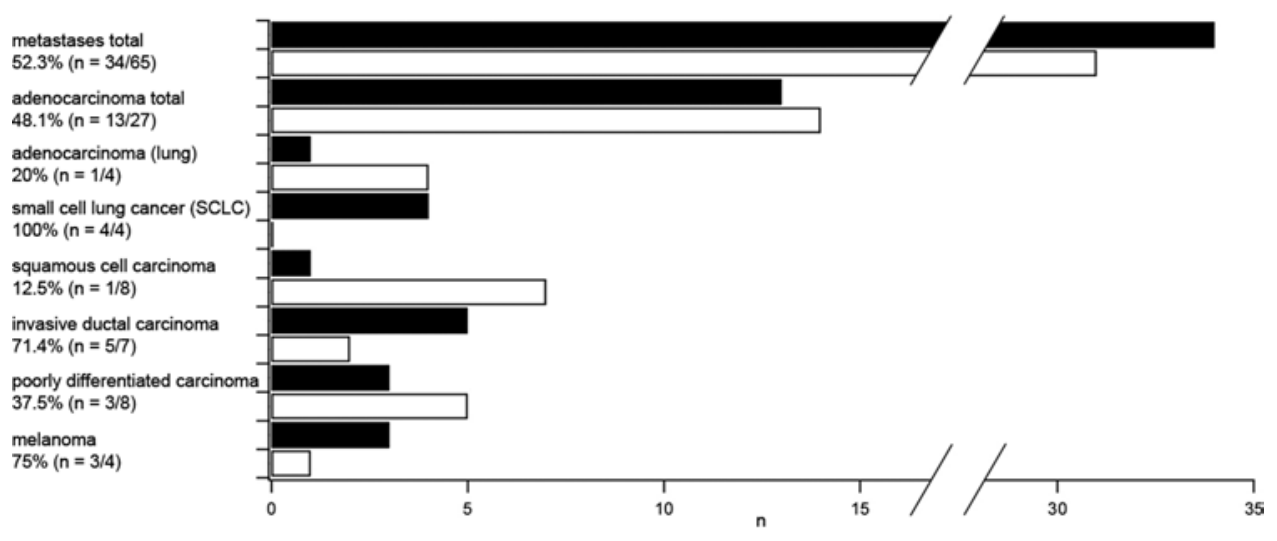

FIG. 5. Graph demonstrating 5-ALA positivity in brain metastases. $\mathrm{n}$ indicates cases with positive fluorescence/total number of cases; black bars = 5-ALA-positive cases; white bars = 5-ALA-negative cases.

gioma tissue and defining the extent of resection in cases with bone, dural, or brain parenchyma invasion. These observations corroborate previous reports that after gross total tumor resection, remnants infiltrating the cortex are difficult to identify under white light inspection and may be visualized only under fluorescence. ${ }^{5}$ Furthermore, intraoperative photodynamic diagnosis provided by 5-ALA facilitates visualization of cranial bone infiltration, 5,19,26 which constitutes an important factor for decreasing the risk for recurrence ${ }^{18}$ and associated poor outcome..$^{13}$ Our study confirms the high rate of 5-ALA fluorescence in meningiomas and therefore highlights the usefulness of intraoperative fluorescence as a routine visual tool for achieving optimal resection.

With respect to their 5-ALA fluorescence behavior, brain metastases form a heterogeneous group; overall positive fluorescence rate was 52\%. The rate of 5-ALA fluorescence was high for gastrointestinal adenocarcinomas (especially of the esophagus and colon), small-cell lung carcinomas, ductal carcinomas of the breast, and melanomas, and fluorescence provided valuable support for visualization of the brain-tumor interface. However, we infrequently observed intrametastatic 5-ALA fluorescence heterogeneity. Others have reported positive 5-ALA fluorescence in peri-tumor areas surrounding brain metastases. ${ }^{20,36,41}$ These reports and our own find- ings indicate that more studies are needed to elucidate the potential role of 5-ALA in improving resection margins in various entities of brain metastasis.

We used 5-ALA fluorescence as an immediate intraoperative indicator of tumor tissue in patients undergoing diagnostic biopsy. In case of 5-ALA-positive sampling, frozen section confirmation was not requested, and the surgical procedure was completed. This approach did not result in any false-negative biopsy results in our case series and made the procedure faster and safer by reducing multiple biopsies and avoiding resampling in a substantial number of patients (67\%). Our findings are consistent with those of von Campe et al., who found that all biopsy samples with positive fluorescence from patients with suspected high-grade gliomas were confirmed as neoplasms. ${ }^{47}$

\section{Conclusions}

Our study confirms previous findings that, in addition to malignant gliomas, rates of positive 5-ALA fluorescence are high in certain brain metastases and meningiomas. In addition, this study shows that the administration of 5-ALA as a surgical adjunct for resection and biopsy of primary brain tumors and brain metastases seems to be safe. In light of the high rate of fluorescence positivity in low-grade glioma WHO I-II $25 \%(n=3 / 12)$

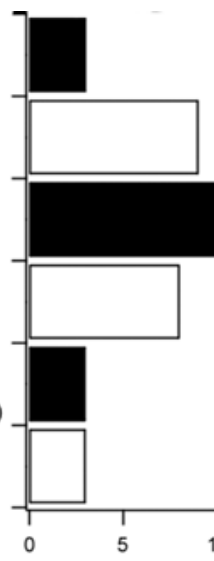

high-grade glioma WHO III-IV $84.6 \%(n=44 / 52)$ diffuse large B-cell lymphoma (DLBCL)
$50 \%(n=3 / 6)$

FIG. 6. Graph showing 5-ALA positivity in brain biopsies. $n$ indicates cases with positive fluorescence/total number of cases; black bars = 5-ALA-positive cases; white bars = 5-ALA-negative cases. 
high-grade gliomas other than glioblastomas, meningiomas, and a variety of metastatic cancers, 5-ALA seems to be a promising tool for enhancing intraoperative identification of tumor tissue and therefore potentially optimizing the extent of resection.

\section{Acknowledgment}

We express our gratitude to Ms. Jeannie Wurz for her editorial support in proofreading the final version of the manuscript.

\section{Disclosure}

The authors report no conflict of interest concerning the materials or methods used in this study or the findings specified in this paper.

The authors have no financial or commercial interest in 5-ALA (Gliolan; medac GmbH, Hamburg, Germany). No funds were or will be received for this study. Financial support for patient care and treatment was provided by Kantonsspital Aarau, Department of Neurosurgery, Aarau, Switzerland.

Author contributions to the study and manuscript preparation include the following. Conception and design: Fandino, Marbacher, Schwyzer. Acquisition of data: Marbacher, Klinger, Nevzati. Analysis and interpretation of data: Marbacher, Klinger, Fischer, Roelcke. Drafting the article: Marbacher, Klinger, Fischer, Diepers. Critically revising the article: Fandino, Marbacher, Schwyzer, Nevzati, Diepers, Roelcke, Fathi, Coluccia. Approved the final version of the manuscript on behalf of all authors: Fandino. Administrative/technical/material support: Fandino. Study supervision: Fandino. Figure preparation: Klinger.

\section{References}

1. Bekelis K, Valdés PA, Erkmen K, Leblond F, Kim A, Wilson $\mathrm{BC}$, et al: Quantitative and qualitative 5-aminolevulinic acidinduced protoporphyrin IX fluorescence in skull base meningiomas. Neurosurg Focus 30(5):E8, 2011

2. Colditz MJ, Jeffree RL: Aminolevulinic acid (ALA)-protoporphyrin IX fluorescence guided tumour resection. Part 1: Clinical, radiological and pathological studies. J Clin Neurosci 19:1471-1474, 2012

3. Colditz MJ, van Leyen K, Jeffree RL: Aminolevulinic acid (ALA)-protoporphyrin IX fluorescence guided tumour resection. Part 2: theoretical, biochemical and practical aspects. J Clin Neurosci 19:1611-1616, 2012

4. Collaud S, Juzeniene A, Moan J, Lange N: On the selectivity of 5-aminolevulinic acid-induced protoporphyrin IX formation. Curr Med Chem Anticancer Agents 4:301-316, 2004

5. Coluccia D, Fandino J, Fujioka M, Cordovi S, Muroi C, Landolt $\mathrm{H}$ : Intraoperative 5-aminolevulinic-acid-induced fluorescence in meningiomas. Acta Neurochir (Wien) 152:1711-1719, 2010

6. Díez Valle R, Tejada Solis S, Idoate Gastearena MA, García de Eulate R, Domínguez Echávarri P, Aristu Mendiroz J: Surgery guided by 5 -aminolevulinic fluorescence in glioblastoma: volumetric analysis of extent of resection in single-center experience. J Neurooncol 102:105-113, 2011

7. Eicker SO, Floeth FW, Kamp M, Steiger HJ, Hänggi D: The impact of fluorescence guidance on spinal intradural tumour surgery. Eur Spine J 22:1394-1401, 2013

8. el-Sharabasy MM, el-Waseef AM, Hafez MM, Salim SA: Porphyrin metabolism in some malignant diseases. Br J Cancer 65:409-412, 1992

9. Eléouet S, Rousset N, Carré J, Bourré L, Vonarx V, Lajat Y, et al: In vitro fluorescence, toxicity and phototoxicity induced by delta-aminolevulinic acid (ALA) or ALA-esters. Photochem Photobiol 71:447-454, 2000

10. Ewelt C, Floeth FW, Felsberg J, Steiger HJ, Sabel M, Langen $\mathrm{KJ}$, et al: Finding the anaplastic focus in diffuse gliomas: the value of Gd-DTPA enhanced MRI, FET-PET, and intraoperative, ALA-derived tissue fluorescence. Clin Neurol Neurosurg 113:541-547, 2011

11. Fotinos N, Campo MA, Popowycz F, Gurny R, Lange N: 5-Aminolevulinic acid derivatives in photomedicine: characteristics, application and perspectives. Photochem Photobiol 82:994-1015, 2006

12. Frei KA, Bonel HM, Frick H, Walt H, Steiner RA: Photodynamic detection of diseased axillary sentinel lymph node after oral application of aminolevulinic acid in patients with breast cancer. Br J Cancer 90:805-809, 2004

13. Gabeau-Lacet D, Aghi M, Betensky RA, Barker FG, Loeffler JS, Louis DN: Bone involvement predicts poor outcome in atypical meningioma. Clinical article. J Neurosurg 111:464471, 2009

14. Hefti M, von Campe G, Moschopulos M, Siegner A, Looser H, Landolt H: 5-aminolevulinic acid induced protoporphyrin IX fluorescence in high-grade glioma surgery: a one-year experience at a single institution. Swiss Med Wkly 138:180-185, 2008

15. Hungerhuber E, Stepp H, Kriegmair M, Stief C, Hofstetter A, Hartmann A, et al: Seven years' experience with 5-aminolevulinic acid in detection of transitional cell carcinoma of the bladder. Urology 69:260-264, 2007

16. Idoate MA, Díez Valle R, Echeveste J, Tejada S: Pathological characterization of the glioblastoma border as shown during surgery using 5-aminolevulinic acid-induced fluorescence. Neuropathology 31:575-582, 2011

17. Ishihara R, Katayama Y, Watanabe T, Yoshino A, Fukushima T, Sakatani K: Quantitative spectroscopic analysis of 5-aminolevulinic acid-induced protoporphyrin IX fluorescence intensity in diffusely infiltrating astrocytomas. Neurol Med Chir (Tokyo) 47:53-57, 2007

18. Jääskeläinen J: Seemingly complete removal of histologically benign intracranial meningioma: late recurrence rate and factors predicting recurrence in 657 patients. A multivariate analysis. Surg Neurol 26:461-469, 1986

19. Kajimoto Y, Kuroiwa T, Miyatake S, Ichioka T, Miyashita M, Tanaka H, et al: Use of 5-aminolevulinic acid in fluorescenceguided resection of meningioma with high risk of recurrence. Case report. J Neurosurg 106:1070-1074, 2007

20. Kamp MA, Grosser P, Felsberg J, Slotty PJ, Steiger HJ, Reifenberger G, et al: 5-aminolevulinic acid (5-ALA)-induced fluorescence in intracerebral metastases: a retrospective study. Acta Neurochir (Wien) 154:223-228, 2012

21. Kennedy JC, Pottier RH: Endogenous protoporphyrin IX, a clinically useful photosensitizer for photodynamic therapy. J Photochem Photobiol B 14:275-292, 1992

22. Kuhnt D, Becker A, Ganslandt O, Bauer M, Buchfelder M, Nimsky C: Correlation of the extent of tumor volume resection and patient survival in surgery of glioblastoma multiforme with high-field intraoperative MRI guidance. Neuro Oncol 13:1339-1348, 2011

23. Laws ER, Parney IF, Huang W, Anderson F, Morris AM, Asher A, et al: Survival following surgery and prognostic factors for recently diagnosed malignant glioma: data from the Glioma Outcomes Project. J Neurosurg 99:467-473, 2003

24. McGirt MJ, Chaichana KL, Gathinji M, Attenello FJ, Than K, Olivi A, et al: Independent association of extent of resection with survival in patients with malignant brain astrocytoma. Clinical article. J Neurosurg 110:156-162, 2009

25. Moriuchi S, Yamada K, Dehara M, Teramoto Y, Soda T, Imakita M, et al: Use of 5-aminolevulinic acid for the confirmation of deep-seated brain tumors during stereotactic biopsy. Report of 2 cases. J Neurosurg 115:278-280, 2011

26. Morofuji Y, Matsuo T, Hayashi Y, Suyama K, Nagata I: Usefulness of intraoperative photodynamic diagnosis using 5-aminolevulinic acid for meningiomas with cranial invasion: technical case report. Neurosurgery 62 (3 Suppl 1):102-104, 2008 
27. Muroi C, Fandino J, Coluccia D, Berkmann S, Fathi AR, Landolt H: 5-Aminolevulinic acid fluorescence-guided surgery for spinal meningioma. World Neurosurg 80:223.e1223.e3, 2012

28. Ohgari Y, Nakayasu Y, Kitajima S, Sawamoto M, Mori H, Shimokawa $\mathrm{O}$, et al: Mechanisms involved in delta-aminolevulinic acid (ALA)-induced photosensitivity of tumor cells: relation of ferrochelatase and uptake of ALA to the accumulation of protoporphyrin. Biochem Pharmacol 71:42-49, 2005

29. Peng Q, Berg K, Moan J, Kongshaug M, Nesland JM: 5-Aminolevulinic acid-based photodynamic therapy: principles and experimental research. Photochem Photobiol 65:235-251, 1997

30. Pichlmeier U, Bink A, Schackert G, Stummer W: Resection and survival in glioblastoma multiforme: an RTOG recursive partitioning analysis of ALA study patients. Neuro Oncol 10: 1025-1034, 2008

31. Ritz R, Feigl GC, Schuhmann MU, Ehrhardt A, Danz S, Noell $S$, et al: Use of 5-ALA fluorescence guided endoscopic biopsy of a deep-seated primary malignant brain tumor. Technical note. J Neurosurg 114:1410-1413, 2011

32. Roberts DW, Valdés PA, Harris BT, Hartov A, Fan X, Ji S, et al: Glioblastoma multiforme treatment with clinical trials for surgical resection (aminolevulinic acid). Neurosurg Clin N Am 23:371-377, 2012

33. Sanai N: Emerging operative strategies in neurosurgical oncology. Curr Opin Neurol 25:756-766, 2012

34. Sanai N, Berger MS: Glioma extent of resection and its impact on patient outcome. Neurosurgery 62:753-766, 2008

35. Sanai N, Snyder LA, Honea NJ, Coons SW, Eschbacher JM, Smith KA, et al: Intraoperative confocal microscopy in the visualization of 5-aminolevulinic acid fluorescence in low-grade gliomas. Clinical article. J Neurosurg 115:740-748, 2011

36. Schucht P, Beck J, Vajtai I, Raabe A: Paradoxical fluorescence after administration of 5-aminolevulinic acid for resection of a cerebral melanoma metastasis. Acta Neurochir (Wien) 153:1497-1499, 2011

37. Stummer W, Nestler U, Stockhammer F, Krex D, Kern BC Mehdorn HM, et al: Favorable outcome in the elderly cohort treated by concomitant temozolomide radiochemotherapy in a multicentric phase II safety study of 5-ALA. J Neurooncol 103:361-370, 2011

38. Stummer W, Novotny A, Stepp H, Goetz C, Bise K, Reulen $\mathrm{HJ}$ : Fluorescence-guided resection of glioblastoma multiforme by using 5-aminolevulinic acid-induced porphyrins: a prospective study in 52 consecutive patients. J Neurosurg 93:1003-1013, 2000

39. Stummer W, Pichlmeier U, Meinel T, Wiestler OD, Zanella F, Reulen HJ: Fluorescence-guided surgery with 5-aminolevulinic acid for resection of malignant glioma: a randomised controlled multicentre phase III trial. Lancet Oncol 7:392-401, 2006

40. Stummer W, Reulen HJ, Meinel T, Pichlmeier U, Schumacher $\mathrm{W}$, Tonn JC, et al: Extent of resection and survival in glioblastoma multiforme: identification of and adjustment for bias. Neurosurgery 62:564-576, 2008
41. Utsuki S, Miyoshi N, Oka H, Miyajima Y, Shimizu S, Suzuki $\mathrm{S}$, et al: Fluorescence-guided resection of metastatic brain tumors using a 5-aminolevulinic acid-induced protoporphyrin IX: pathological study. Brain Tumor Pathol 24:53-55, 2007

42. Utsuki S, Oka H, Sato S, Suzuki S, Shimizu S, Tanaka S, et al: Possibility of using laser spectroscopy for the intraoperative detection of nonfluorescing brain tumors and the boundaries of brain tumor infiltrates. Technical note. J Neurosurg 104:618-620, 2006

43. Valdes PA, Bekelis K, Harris BT, Wilson BC, Leblond F, Kim A, et al: 5-Aminolevulinic-acid-induced protoporphyrin ix fluorescence in meningioma: qualitative and quantitative measurements in vivo. Neurosurgery [epub ahead of print], 2013

44. Valdés PA, Kim A, Brantsch M, Niu C, Moses ZB, Tosteson $\mathrm{TD}$, et al: $\delta$-aminolevulinic acid-induced protoporphyrin IX concentration correlates with histopathologic markers of malignancy in human gliomas: the need for quantitative fluorescence-guided resection to identify regions of increasing malignancy. Neuro Oncol 13:846-856, 2011

45. Valdés PA, Leblond F, Kim A, Harris BT, Wilson BC, Fan XY, et al: Quantitative fluorescence in intracranial tumor: implications for ALA-induced PpIX as an intraoperative biomarker. Clinical article. J Neurosurg 115:11-17, 2011

46. Valdés PA, Moses ZB, Kim A, Belden CJ, Wilson BC, Paulsen $\mathrm{KD}$, et al: Gadolinium- and 5-aminolevulinic acid-induced protoporphyrin IX levels in human gliomas: an ex vivo quantitative study to correlate protoporphyrin IX levels and blood-brain barrier breakdown. J Neuropathol Exp Neurol 71:806-813, 2012

47. von Campe G, Moschopulos M, Hefti M: 5-Aminolevulinic acid-induced protoporphyrin IX fluorescence as immediate intraoperative indicator to improve the safety of malignant or high-grade brain tumor diagnosis in frameless stereotactic biopsies. Acta Neurochir (Wien) 154:585-588, 2012

48. Widhalm G, Wolfsberger S, Minchev G, Woehrer A, Krssak M, Czech T, et al: 5-Aminolevulinic acid is a promising marker for detection of anaplastic foci in diffusely infiltrating gliomas with nonsignificant contrast enhancement. Cancer 116:1545-1552, 2010

49. Zhao S, Wu J, Wang C, Liu H, Dong X, Shi C, et al: Intraoperative fluorescence-guided resection of high-grade malignant gliomas using 5-aminolevulinic acid-induced porphyrins: a systematic review and meta-analysis of prospective studies. PLOS ONE 8:e63682, 2013

Manuscript submitted: October 13, 2013

Accepted: December 13, 2013.

Please include this information when citing this paper: DOI: 10.3171/2013.12.FOCUS13464.

Address correspondence to: Javier Fandino, M.D., c/o Editorial Office, Department of Neurosurgery, Kantonsspital Aarau, Aarau CH-5000, Switzerland. email: neurosurgery@ksa.ch. 